\title{
COMPARISON OF THE APPROXIMATE SOLUTION WITH THE SOLUTION OF THE DIFFERENTIAL EQUATION FOR THE STATIC PROBLEM OF DEEP-PROFILED SANDWICH PANELS
}

\author{
Jolanta Pozorska \\ Department of Mathematics, Czestochowa University of Technology, Poland \\ jolanta.pozorska@im.pcz.pl
}

Received: 10 September 2020; Accepted: 5 December 2020

\begin{abstract}
The article presents an approximate method of solving the problem of statics of sandwich elements with deep-profiled facings. This method is compared with the widely accepted theory of sandwich beams. The example of uniformly loaded, simply-supported, single-span sandwich beam is presented. The results of displacements, internal forces and stresses obtained by both methods are compared. The results confirm the good compliance of the presented solutions, in particular in terms of displacements.
\end{abstract}

MSC 2010: 65C20, 68U07, 68U20, 74K20

Keywords: approximate solutions, differential equations, sandwich panels, static problem

\section{Introduction}

This paper considers the problem of the statics of a sandwich panel. Sandwich panels have been the subject of research and scientific analysis for many years. These structures belong to the group of layered composites, and the enormous diversity of the properties of the facings and the core materials makes it necessary to create proper models of such a structure, taking into account various phenomena and failure mechanisms. Due to a fairly simple but very effective structure, sandwich panels have undeniable advantages that are used in practice. Thin but rigid and resistant to external environmental influences, the facings of the sandwich panel transmit bending. A thick but light core transfers shear, stabilizes the facings and provides an appropriate distance between the facings, which affects the stiffness and strength of the panel. The core is often also an excellent thermal insulation, but it can fulfill other functions.

The problem of the statics of sandwich panels was intensively analyzed in the middle of the 20th century. The various theories were mainly developed in two powerful research centers in the United States: The National Advisory Committee for Aeronautics (NACA - NASA at present) and Forest Product Laboratories of the United States Forest Service. These theories, often very complex, turned out to 
be unattractive from the point of view of practicing engineers. The author of the theory known as The Ordinary Sandwich Panel Theory (OSAPT), which is applied in practice, is Nicholas John Hoff who considered the strain energy and derived the governing equations for an isotropic sandwich panel with thick faces [1]. This theory was disseminated through the monographs of Plantema [2], Allen [3], as well as Stamm and Witte [4]. In 2001, the monograph [5] was published under the auspices of Working Commission W056, which led to the creation of the European Code 14509 [6]. Of course, the EC is based on the OSAPT.

Despite such an orderly situation in terms of the applied theory, sandwich panels still arouse considerable interest [7]. This is due, among other things, to the search for solutions that will be useful in numerical applications. For this reason, various finite elements are created or new approximate solutions are sought $[8,9]$. The aim of this study is to compare the known approximate solution [5] with the classic OSAPT solution for sandwich panels with deep-profiled facing. The comparison will be made for a single-span sandwich panel uniformly loaded along its entire length.

\section{Description of the problem and classical solution}

According to [6], under typical support conditions, sandwich panels are treated as beam elements. One considers a simply supported one-span beam with a span $L$ (Fig. 1). The cross-section of the beam consists of two facings and a core, with the upper facing assumed to be deep-profiled (Fig. 1). This is a typical case of a structure used as a roof covering. The $G_{C}$ and $A_{C}$ denote shear modulus and the cross-sectional area of the core. The term $B_{S}$ corresponds to the bending stiffness of the facings with respect to the global center line of the sandwich panel, whereas $B_{D}$ represents the sum of the bending stiffness of the facings with respect to their own center lines $\left(B_{D}=B_{F 1}+B_{F 2}\right)$. The total bending stiffness of the panel is $B=B_{S}+B_{D}$. Symbol $q$ denotes the distributed transverse load. Subscripts 1 and 2 correspond to the lower and upper facings.

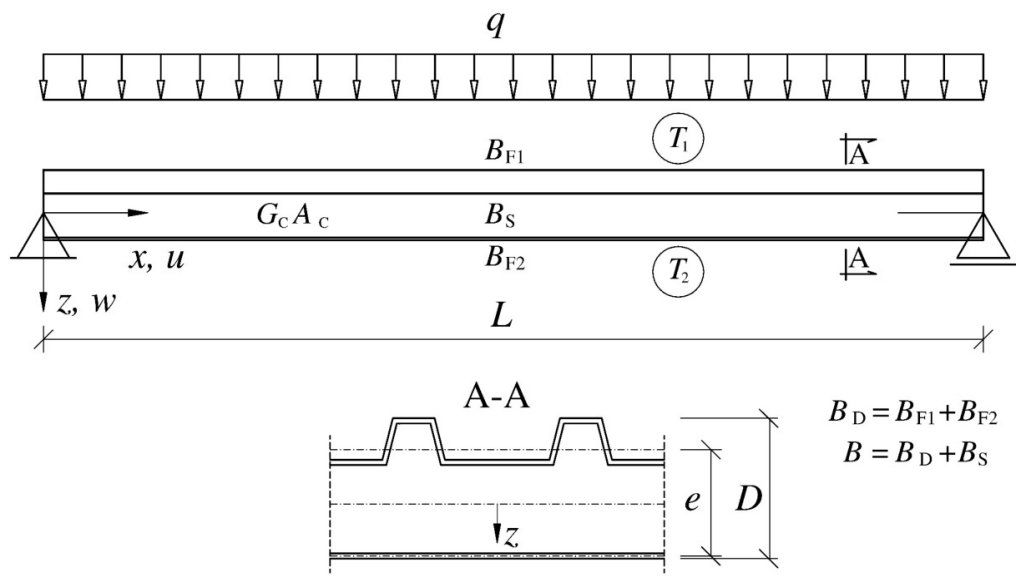

Fig. 1. The static system of a one-span, deep-profiled sandwich panel 
The structural behavior of a sandwich beam can be described by two differential equations:

$$
\begin{gathered}
-\frac{B_{D}}{G_{C} A_{C}} \cdot w^{I V}+\frac{B_{S}+B_{D}}{B_{S}} \cdot w^{\prime \prime}=-\frac{M}{B_{S}}-\frac{q}{G_{C} A_{C}}-\theta, \\
-\frac{B_{D}}{G_{C} A_{C}} \cdot \gamma^{\prime \prime}+\frac{B_{S}+B_{D}}{B_{S}} \cdot \gamma=\frac{V}{G_{C} A_{C}}-\frac{B_{D}}{G_{C} A_{C}} \cdot \theta^{\prime} .
\end{gathered}
$$

where transversal deflection $w$ and shear strain $\gamma$ are the functions of the position coordinate $x$. Symbol $\theta$ denotes an initial curvature induced by a temperature difference between facings $\left(T_{2}-T_{1}\right)$. Terms $M$ and $V$ denote the bending moment and shear force, respectively. The quantities representing loads or internal forces $(q, \theta, M, V)$ generally depend on the position variable $x$.

The classical solution of (1)-(2) was given by many authors, and some discussion on the solution was presented in [9]. The solution for the case of a uniform load $q$ is:

$$
\begin{gathered}
w(x)=\frac{q L^{4}}{B}\left[\frac{1}{24} \xi\left(1-2 \xi^{2}+\xi^{3}\right)+\frac{1}{2 \alpha \lambda^{2}} \xi(1-\xi)-\frac{1}{\alpha \lambda^{4}} \frac{\cosh (\lambda / 2)-\cosh (\lambda(1-2 \xi) / 2)}{\cosh (\lambda / 2)}\right] \\
\gamma(x)=\frac{q L^{3}}{B} \beta\left[\frac{1}{2}(1-2 \xi)-\frac{1}{\lambda} \frac{\sinh (\lambda(1-2 \xi) / 2)}{\cosh (\lambda / 2)}\right]
\end{gathered}
$$

where $\xi=x / L$ ( $L$ is the span of the beam). The symbols $\alpha, \beta, \lambda$ represent the following dimensionless auxiliary quantities:

$$
\begin{gathered}
\alpha=\frac{B_{F 1}+B_{F 2}}{B_{S}}=\frac{B_{D}}{B_{S}}, \\
\beta=\frac{B_{S}}{G_{C} A_{C} L^{2}}, \\
\lambda^{2}=\frac{1+\alpha}{\alpha \beta} .
\end{gathered}
$$

\section{Approximate solution}

Hyperbolic functions may cause some numerical difficulties in certain situations. No wonder then that there was a proposal for an approximate solution of the presented problem [5]. It was assumed in the approximate approach that the applied 
load is shared between two separate load-carrying systems: the sandwich part which includes the influence of the core shear, and the flange part which purely involves bending of flanges. The two systems are assumed to be completely independent, with the proviso that at some point (usually in the middle of the span), the displacement of the two systems should be identical.

In the case of a simply-supported beam carrying a uniformly distributed load $q$, the deflections at mid-span of the two parts (sandwich and flange) can be presented as:

$$
\begin{gathered}
w_{S}=\frac{5}{384} \frac{q_{S} L^{4}}{B_{S}}(1+k), \\
w_{D}=\frac{5}{384} \frac{q_{D} L^{4}}{B_{D}},
\end{gathered}
$$

where $q_{S}$ and $q_{D}$ represent load components carried by the sandwich and flange part, respectively. Symbol $k$ denotes the shear factor (the same as in the case of classical solution), which in the analyzed case is:

$$
k=\frac{9.6 B_{S}}{G_{C} A_{C} L^{2}} .
$$

Using the obvious relationship $q=q_{S}+q_{D}$ we can express the loads as:

$$
\begin{gathered}
q_{S}=q(1-\delta), \\
q_{D}=q \delta .
\end{gathered}
$$

with

$$
\delta=\frac{(1+k) B_{D}}{B_{S}+(1+k) B_{D}} .
$$

As a result of this approach, the deflections and stresses at any point can be determined as for the simple (Bernoulli) beam theory. For example, the deflection in the middle of the span will be:

$$
w_{\max }=\frac{5}{384} \frac{q L^{4}}{B_{S}}(1-\delta)(1+k),
$$

and the extreme stresses in the compressed (upper) facing:

$$
\sigma_{F 11}=\frac{q L^{2}}{8}\left(\frac{\delta}{I_{F 1}} z_{F 1}+\frac{(1-\delta)}{e A_{F 1}}\right),
$$


where: $I_{F 1}$ - moment of inertia of the upper facing, $A_{F 1}$ - area of the cross-section of the upper facing, $e$ - distance between the centers of gravity of the facings, $z_{F 1}$ - distance to the extreme fibers of the deep-profiled cross-section.

\section{Comparison of the exact and approximate solutions}

A comparison for an exemplary static system is provided below. The 'exact' solution should be understood as the solution resulting from the system of differential equations (1)-(2). It is an analytical solution, which is true for the assumed theory. The approximate solution is expressed in equations (8)-(15).

The comparison of solutions is presented for a typical sandwich panel with the following geometric and material parameters (cf. Fig. 1): $D=0.12 \mathrm{~m}, d=0.08 \mathrm{~m}$, $A_{F 1}=0.000551 \mathrm{~m}^{2}, I_{F 1}=9.792 \mathrm{e}-8 \mathrm{~m}^{4}, E_{F 1}=210 \mathrm{GPa}, A_{F 2}=0.000483 \mathrm{~m}^{2}, I_{F 2}=$ $=8.517 \mathrm{e}-12 \mathrm{~m}^{4}, E_{F 2}=210 \mathrm{GPa}, t_{F 1}=0.00046 \mathrm{~m}, t_{F 2}=0.00046 \mathrm{~m}, e=0.0853 \mathrm{~m}$, $G_{C}=3.4 \mathrm{MPa}, A_{C}=0.0853 \mathrm{~m}^{2}$. A sandwich panel (beam) with a span of $L=5.0 \mathrm{~m}$ is uniformly loaded with $q=1.0 \mathrm{kN} / \mathrm{m}$. In this case one obtaines: $B_{S}=393.27 \mathrm{kNm}$, $B_{D}=20.565 \mathrm{kNm}^{2}, \alpha=0.05229, \beta=0.05087, \lambda=19.8892, k=0.1526$. The load $q$ is divided according to $\delta=0.0722$, therefore $q_{S}=0.9278 \mathrm{kN} / \mathrm{m}, q_{D}=0.0722 \mathrm{kN} / \mathrm{m}$.

The results of comparison are presented in Figures 2-5, namely the difference in the total displacement between the approximate and analytical solution (the 'exact' deflection is $w=0.02861 \mathrm{~m}$ ), the relative error of the total displacement, the relative error of the extreme normal stress in the upper facing, and the relative error of the shear force components. Note that the values with the superscript $a$ correspond to the approximate solution and the values without the superscript correspond to the analytical solution (OSAPT).

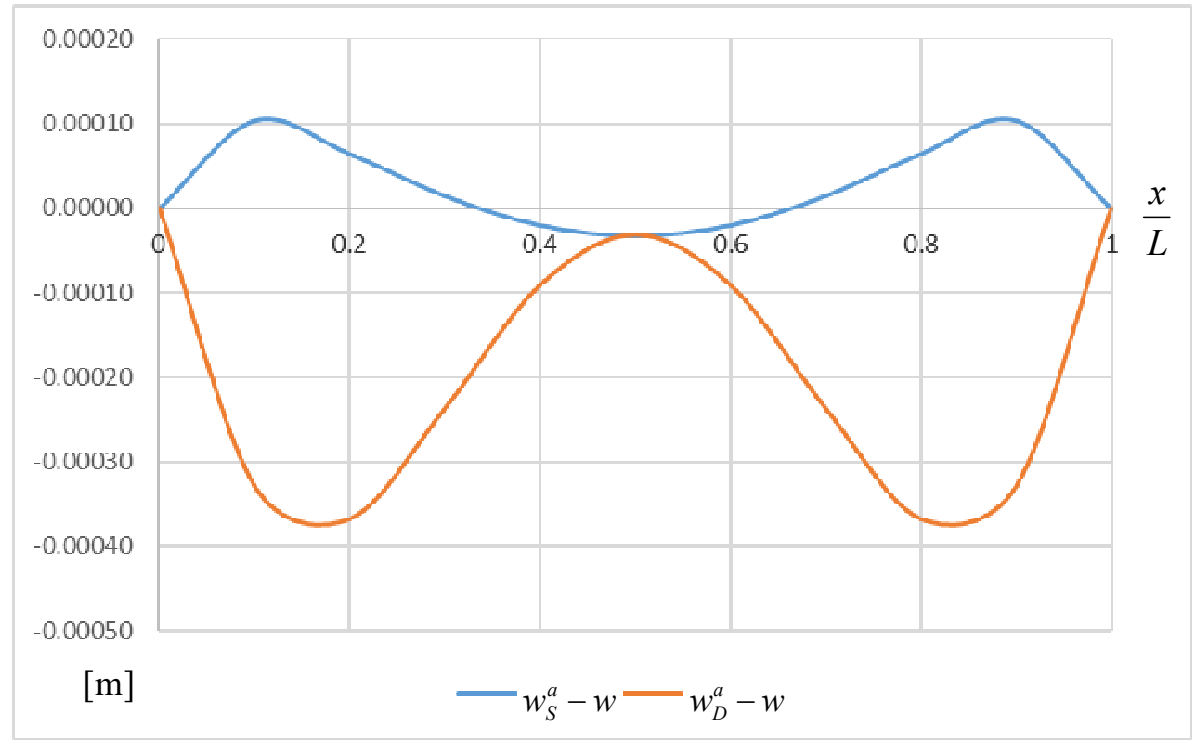

Fig. 2. The difference in the total displacement between approximate and analytical solution 


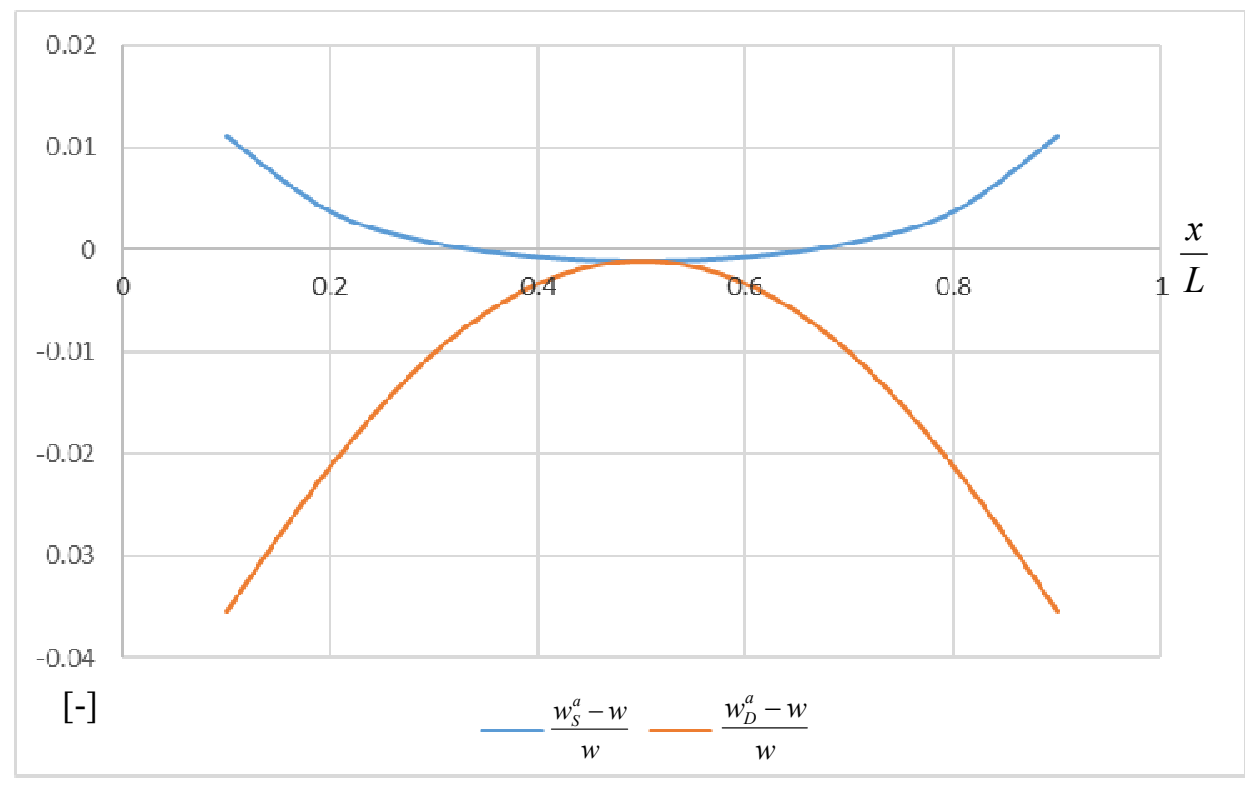

Fig. 3. The relative error of the total displacement

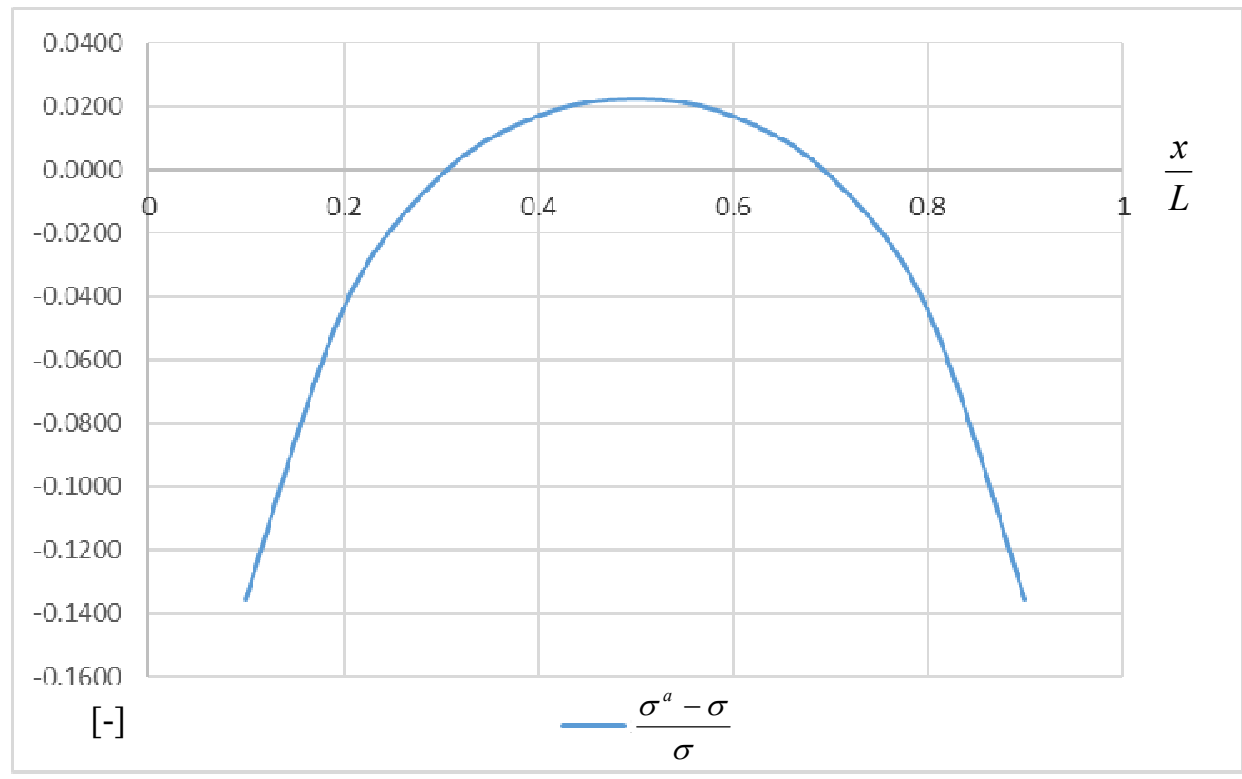

Fig. 4. The relative error of the extreme normal stress in the upper facing 


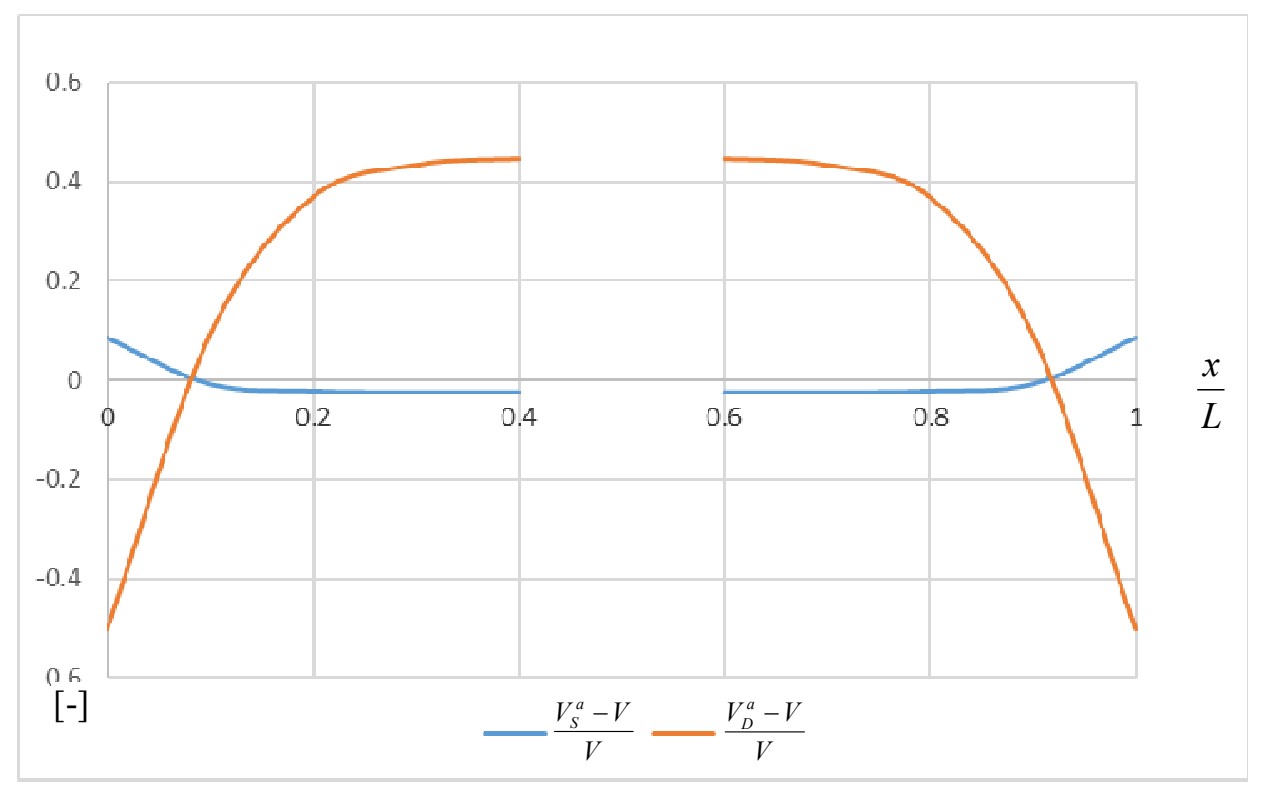

Fig. 5. The relative error of the shear force components

By analyzing the presented graphs, it can be concluded that the presented approximate solution is close to the solution according to OSAPT. The difference in displacement of a $5.0 \mathrm{~m}$ span beam at any point of the beam does not exceed $0.4 \mathrm{~mm}$, and the relative error does not exceed $4 \%$. It is worth noting that the displacements of the sandwich part differ from the displacements of the flange part, but this is a consequence of the adopted simplifications. Only in the middle of the span are the displacements of both parts identical (see Figs. 2 and 3). The accuracy of the obtained normal stresses in the upper facing looks much worse. In the middle of the span, the relative error amounts to $2 \%$, while in the vicinity of the supports it is in the order of several percent (Fig. 4). Fortunately, the extreme stresses for this case are achieved in the middle of the span. In the case of deep-profile panels, the shear force is transmitted through the core $\left(V_{S}\right)$ and deep-profiled facing $\left(V_{D}\right)$. For the presented example, the relative error of $V_{S}$ is the highest at the support and amounts to $8.5 \%$. The greatest relative error concerns $V_{D}$ and amounts to as much as $50.3 \%$ at supports.

Let us also explain why the graphs presented in Figures 3-5 do not cover the entire range of the variable $x$. The displacement of the beam on the support is zero, which means that the relative error is expressed by the indefinite symbol $0 / 0$. Similarly, with normal stresses at the support, the stress depends directly on the bending moment, which equals zero for $x=0$ and $x=L$. The same applies to the shear force, which in the middle of the beam span is equal to zero. 


\section{Conclusions}

The paper compares the analytical solution of the classic theory (OSAPT) with the approximate solution in which the sandwich part was artificially separated from the flange part. Such a procedure, quite common in engineering, allows for a simple and quick solution to the problem of the statics of sandwich beams. The approximate approach has proved to be successful and the accuracy of the approximate displacements was surprisingly good. Unfortunately, some concerns are raised by the inaccuracy of the obtained bending moments and normal stresses, although fortunately these inaccuracies mainly relate to the support zones, where normal stresses do not play a key role. The situation will certainly change in the case of multi-span systems, because in this case extreme moments and stresses most often occur at the supports. Even greater caution is advised when using the approximate method to determine the shear effects. The $8.5 \%$ difference in value may be decisive in assessing the safety of a sandwich panel [11].

Although the paper does not present a suitable example, it should be added that the additional analysis show that the relative errors of the approximate approach are the greater the shorter the beam. After all, the approximate solution is an example of interesting creativity in solving mechanical problems (in this case layered beams). Moreover, the presented analysis leads to a certain cautiousness in the evaluation of the applied calculation methods.

\section{References}

[1] Hoff, N.J., \& Mautner, S.E. (1948). Bending and buckling of sandwich beams. Journal of Aeronautical Sciences, 15(12), 707-720.

[2] Plantema, F.J. (1966). Sandwich Construction. John Wiley \& Sons, Inc.

[3] Allen, H.G. (1969). Analysis and Design of Structural Sandwich Panels. Oxford: Pergamon Press Ltd.

[4] Stamm, K., \& Witte, H. (1974). Sandwichkonstruktionen - Berechnung, Fertigung, Ausführung. Springer-Verlag.

[5] Davies, J.M. (2001). Lightweight Sandwich Construction. Oxford: Blackwell Science.

[6] EN 14509. Self-supporting skin metal faced insulating panels - Factory made products - Specifications, 2013.

[7] Chuda-Kowalska, M., \& Malendowski, M. (2016). The influence of rectangular openings on the structural behaviour of sandwich panels with anisotropic core. Journal of Applied Mathematics and Computational Mechanics, 15(3), 15-25.

[8] Carrera, E., \& Brischetto, S. (2009). A Survey with numerical assessment of classical and refined theories for the analysis of sandwich plates. Applied Mechanics Reviews, 62(1), 010803, 1-17, DOI: 10.1115/1.3013824.

[9] Vijayakumar, K. (2013). On uniform approximate solutions in bending of symmetric laminated plates. Computers, Materials, \& Continua, 34, 1, 1-25.

[10] Błaszczuk, J., \& Pozorski, Z. (2011). Engineering aspects of the mathematical model of deep-profiled sandwich panel. Scientific Research of the Institute of Mathematics and Computer Science, 2(10), 31-42.

[11] Pozorska, J., \& Pozorski, Z. (2017). Analysis of the failure mechanisms of the sandwich panel at the support. Procedia Engineering, 177, 168-174. 